\title{
Comparison of lesion level and ischemic modified albumin in peripheral artery disease
}

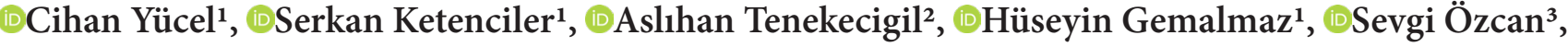 \\ №nihan Kayalar ${ }^{1}$ \\ ${ }^{1}$ Prof. Dr. Cemil Taşçıŏlu City Hospital, Department of Cardiovascular Surgery, İstanbul, Turkey \\ ${ }^{2}$ Bağcllar Training and Research Hospital, İstanbul, Turkey \\ ${ }^{3}$ Bağcllar Training and Research Hospital, Department of cardiology, İstanbul, Turkey
}

Cite this article as: Yücel C, Ketenciler S, Tenekecigil A, Gemalmaz H, Özcan S, Kayalar N. Comparison of lesion level and ischemic modified albumin in peripheral artery disease. Anatolian Curr Med J 2021; 3(3); 204-207.

\begin{abstract}
Aim: Research is ongoing to discover biomarkers that can be used to monitor disease progression and predict prognosis in peripheral artery disease (PAD). Ischemia-modified albumin (IMA) is known to predict the risk for PAD. The aim of this study was to investigate whether serum IMA levels were associated with PAD lesions and ankle-brachial index (ABI) values, and to determine the accuracy of IMA levels in predicting PAD prognosis.

Material and Method: Seventy-three patients with PAD who applied to our hospital between July and September 2018 were included in the study. The lesion levels of patients with an ankle-brachial index (ABI) value lower than or equal to 0.9 were determined by computed tomography angiography (CTA). Patients were grouped according to their lesion levels and Fontaine classification. Serum IMA concentrations were measured and compared with regard to lesion levels and ABI values.

Results: Sixty-five of the patients (89\%) were male. The mean age of the patients was $62.2 \pm 8.8$ (43-77) years. The mean serum IMA level was $25.9 \pm 26.7(10-218) \mathrm{ng} / \mathrm{ml}$. Serum IMA levels were found to be lower in patients with bilateral iliac artery lesions $(\mathrm{p}=0.040)$. There was no statistically significant relationship between other parameters and serum IMA levels ( $p>0.05$ for all). Conclusion: There is a need for biomarkers that can predict lesion level and prognosis in patients with PAD. Although no statistically significant relationship was found between lesion level and serum IMA concentrations in this study, further studies involving a higher number of patients may provide more informative data on this subject.
\end{abstract}

Keywords: Ischemia-modified albumin, peripheral artery disease, ankle-brachial index

\section{INTRODUCTION}

Since there is no specific laboratory test for the diagnosis of peripheral artery disease (PAD), imaging techniques remain the only option for diagnosis. Advanced imaging techniques, such as magnetic resonance imaging angiography, computed tomography angiography (CTA) or digital subtraction angiography can be used in the diagnosis of PAD (1).

Ankle-brachial index (ABI) is used in the clinical followup of the patients with PAD. ABI measurements have a sensitivity of $95 \%$ and a specificity of $99 \%$ in patients with angiography-diagnosed $\mathrm{PAD}$, as determined by $\mathrm{ABI}$ values lower than or equal to 0.9 which are diagnostic for PAD (reference range: 1.0-1.3) (2). Nevertheless, studies continue to reveal biomarkers that can be used to monitor treatment and predict prognosis in PAD. One of these markers is ischemia-modified albumin (IMA), which is has also been shown to be associated with risk for ischemia, oxidative stress and cardiovascular diseases $(3,4)$. Besides ABI, IMA may be helpful to predict the risk for PAD and could be used in the early diagnosis of PAD (4). Various studies have reported that high serum IMA levels were found in patients with PAD $(5,6)$.

The aim of this study is to investigate IMA concentrations in PAD and its association with PAD lesion level and ABI value. We also sought to assess whether IMA concentrations could predict PAD prognosis.

\section{MATERIAL AND METHOD}

Seventy-three patients with PAD who applied to our hospital between July and September 2018 were included in the study. The study was carried out with the permission of Clinical Researches Ethics Committee 
of Okmeydanı Training and Research Hospital (Date: 19.06.2018, Decision No: 48670771-514.10). Informed consent was obtained from all patients. All procedures were carried out in accordance with the ethical rules and the principles of the Declaration of Helsinki.

Patients with a history of angina pectoris, myocardial infarction or heart failure within the last six months were excluded from the study. None of the patients included in the study had a history of ischemic stroke, transient ischemic attack, coronary artery spasm, or renal failure.

The ABI values of all patients were measured. The lesion levels of the patients with an ankle-brachial index (ABI) value of $\leq 0.9$ was determined by computed tomography angiography (CTA). The patients were grouped according to the Fontaine classification and also lesion levels. Three groups were formed with regard to lesion levels: iliac artery, knee joint and below the knee. In addition, multiple lesions were assessed with regard to their localization (only a single site, two of the defined sites, or three sites), as well as their unilateral or bilateral presence.

Peripheral blood samples of the patients were taken into biochemistry tubes and centrifuged to obtain serum. The serum IMA levels were made with Albumin Cobalt Binding Test. This test is based on the colorimetric measurement of the colored complex formed by dithiothreitol (DTT) and free cobalt (the remaining amount that has not been bound to albumin due to the presence of IMA) (7). The materials used were as follows: cobalt chloride (0.1\%), DTT $(1.5 \mathrm{mg} /$ $\mathrm{ml})$ and $\mathrm{NaCl}(0.9 \%)$ solutions, glass tubes, a vortex, an adjustable automatic Eppendorf pipette, disposable plastic micro cuvettes and a spectrophotometer. After adding 50 $\mu \mathrm{l}$ of cobalt chloride $(0.1 \%)$ to $200 \mu \mathrm{l}$ patient serum, it was vortexed and incubated for 10 minutes to allow albumincobalt binding to occur. Then, $50 \mu \mathrm{LTT}(1.5 \mathrm{mg} / \mathrm{mL})$ was added and incubated for 2 minutes. After the incubation, the reaction was quenched by adding $1 \mathrm{~mL}$ of $\mathrm{NaCl}(0.9 \%)$ to the mixture. Distilled water was used instead of DTT for the sample blank. The differences between the absorbance values of patient samples and sample blank read at $470 \mathrm{~nm}$ in the spectrophotometer were recorded as serum IMA levels.

The Statistical Package for the Social Sciences (SPSS, IBM, version 20.0) program was used for the analysis of all data. Sociodemographic characteristics of the study groups were presented in descriptive statistical expressions such as, number, percentage and standard deviation. Quantitative data were described with mean, standard deviation and minimum and maximum values. The compliance of quantitative data to normal distribution was determined by the Shapiro-Wilk test. The Mann-Whitney U test and Spearman's correlation coefficient were used for the analyses.

\section{RESULTS}

Eight of the patients (11\%) were female, and $65(89 \%)$ were male. The mean age of the patients was $62.2 \pm 8.8$ (43-77) years. Forty-three (58.9\%) had comorbid diabetes mellitus. The distribution of demographic characteristics and history of the study groups are presented in Table 1.

The present study investigated the relationship between PAD lesion level and serum IMA level. No significant correlation was found between patients' medical histories, demographic or clinical characteristics and serum IMA levels ( $\mathrm{p}>0.05$ for all) (Table 2).

The mean serum IMA levels of the patients were $25.9 \pm 26.7(10-218) \mathrm{ng} / \mathrm{ml}$. Serum IMA levels were found to be lower in patients with bilateral iliac artery lesions $(p=0.040)$. There was no statistically significant relationship between serum IMA concentrations and ABI values, Fontaine classification ( $p>0.05$ for each) (Table 3).

\begin{tabular}{|lcc|}
\hline Table 1. Preoperative data of patients & \multicolumn{2}{l|}{} \\
\hline Age (years) & $62.2 \pm 8.8$ & $43-77$ \\
\hline Variable & $\mathbf{n}$ & \% of total \\
\hline Sex & & \\
Male & 65 & 89 \\
Female & 8 & 11 \\
Hypertension & 28 & 38.4 \\
Smoking & 59 & 80.8 \\
Diabetes mellitus & 43 & 58.9 \\
Coronary Artery Disease & 29 & 39.7 \\
Concomitant CABG & 8 & 11 \\
COPD & 3 & 4.1 \\
Hyperlipidemia & 19 & 26 \\
Carotid stenosis & 2 & 2.7 \\
\hline COPD: Chronic obstructive pulmonary disease, CABG: Coronary artery bypass graft \\
\hline
\end{tabular}

\section{DISCUSSION}

High serum IMA levels were found in patients with PAD (5). However, to our knowledge, there are no studies investigating the relationship between PAD lesion level and IMA concentration in the literature. The presence of diffuse lesions in patients with PAD is an indicator of poor prognosis (8).

In a study by Özkan et al. (8), severe ischemia was not observed in patients with iliac artery involvement, whereas severe foot ischemia was observed in patients with below-knee vascular lesions. This result may suggest that ischemia is relatively mild when artery stenosis is anatomically superior, possibly due to the presence of better collateral perfusion in this area. Further studies involving more patients are needed to reach a definitive conclusion on this subject. 
Table 2. Distribution of IMA values of the study group according

\begin{tabular}{|c|c|c|c|}
\hline & $\mathbf{n}(\%)$ & $\begin{array}{c}\text { Mean } \\
\text { IMA }(\mathbf{n g} / \mathrm{ml})\end{array}$ & $\mathbf{p}$ \\
\hline \multicolumn{4}{|l|}{ Age (years) } \\
\hline$<65 y 11$ & $38(52.1)$ & $29 \pm 36$ & 1.110 \\
\hline$>65 y_{11}$ & $35(47.9)$ & $23 \pm 7$ & 0.267 \\
\hline \multicolumn{4}{|l|}{ Sex } \\
\hline Male & $65(89.0)$ & $26 \pm 28$ & 0.118 \\
\hline Female & $8(11)$ & $27 \pm 10$ & 1.563 \\
\hline Hypertension & $28(38.4)$ & $29 \pm 38$ & 0.901 \\
\hline Smoking & $59(80.9)$ & $26 \pm 29$ & 0.492 \\
\hline Diabetes mellitus & $43(58.9)$ & $22 \pm 8$ & 0.766 \\
\hline Coronary Artery Disease & $29(39.7)$ & $21 \pm 7$ & 0.401 \\
\hline Concomitant CABG & $8(11)$ & $22 \pm 8$ & 0.839 \\
\hline COPD & $3(4.1)$ & $23 \pm 9$ & 0.824 \\
\hline Hyperlipidemia & $19(26)$ & $21 \pm 7$ & 0.458 \\
\hline Carotid stenosis & $2(2.7)$ & $16 \pm 5$ & 0.129 \\
\hline Iliac artery stenosis (right) & $9(12.3)$ & $19 \pm 5$ & 0.182 \\
\hline Iliac artery stenosis (left) & $13(17.8)$ & $28 \pm 30$ & 0.449 \\
\hline SFA (right) & $27(37)$ & $22 \pm 8$ & 0.775 \\
\hline SFA (left) & $32(43.8)$ & $30 \pm 35$ & 0.103 \\
\hline BTK(right) & $3(4.1)$ & $21 \pm 4$ & 0.865 \\
\hline BTK(left) & $4(5.5)$ & $22 \pm 3$ & 0.861 \\
\hline Only unilateral BTK & $7(9.6)$ & $22 \pm 8$ & 0.822 \\
\hline Only bilateral BTK & $4(5.5)$ & $22 \pm 3$ & 0.842 \\
\hline Only unilateral iliac artery & $8(11)$ & $34 \pm 37$ & 0.578 \\
\hline Only bilateral iliac artery & $4(5.5)$ & $16 \pm 3$ & 0.040 \\
\hline Only unilateral SFA & $19(26)$ & $33 \pm 46$ & 0.669 \\
\hline Only bilateral SFA & $5(6.8)$ & $20 \pm 5$ & 0.824 \\
\hline Iliac artery and SFA & $4(5.5)$ & $22 \pm 5$ & 0.752 \\
\hline Iliac artery and BTK & $1(1.4)$ & 12 & 0.137 \\
\hline SFA and BTK & $21(28.8)$ & $24 \pm 8$ & 0.534 \\
\hline Single level lesion & $47(64.4)$ & $28 \pm 33$ & 0.708 \\
\hline Lesion on two levels & $26(35.6)$ & $23 \pm 8$ & 0.708 \\
\hline Bilateral lesion & $24(32.9)$ & $22 \pm 8$ & 0.842 \\
\hline Unilateral lesion & $49(67.1)$ & $28 \pm 32$ & 0.842 \\
\hline
\end{tabular}

\begin{tabular}{|c|c|c|}
\hline & \multicolumn{2}{|c|}{ IMA } \\
\hline & $\mathbf{r}$ & $\mathbf{p}$ \\
\hline Walking distance & -0.103 & 0.386 \\
\hline $\mathrm{ABI}$ & -0.081 & 0.498 \\
\hline
\end{tabular}

In this study, patients with iliac artery lesions, those with above-knee and those with below-knee vascular lesions were grouped separately. There was no statistically significant relationship between vascular lesion levels and IMA concentrations ( $p>0.05$ for all). However, the serum IMA levels of patients with bilateral iliac artery lesions were found to be significantly lower $(\mathrm{p}=0.040)$. This result may indicate that skeletal muscle ischemia is relatively mild in patients with isolated proximal stenosis, which could again be associated with greater collateral perfusion in this area.
When early diagnosis is not made and appropriate care is not provided, patients with PAD may develop ischemia that ultimately leads to limb amputation and increased morbidity and mortality.

It has recently been reported that the atherosclerotic process in the lower extremities in PAD is not only limited to causing vascular stenosis, but also affects microvascular endothelial cell activity and metabolism along with skeletal muscle microvascular flow reservoir (9). Microvascular endothelial cell activity and skeletal muscle microvascular flow were found to be reduced significantly in patients with PAD in both upper and lower extremities $(10,11)$. The pathophysiological explanation of the relationship between PAD and IMA is that the decrease in lower extremity perfusion and oxygenation in patients with PAD causes microvascular dysfunction and triggers albumin modification.

Transformation of albumin to IMA due to ischemic effects results in decreased binding capacity of cobalt, copper and nickel to the $\mathrm{N}$-terminal region of albumin. Therefore, the change in IMA level in the presence of ischemia has been investigated frequently in recent years.

Various studies reported that serum IMA levels increased in patients with pulmonary embolism, those that underwent cardiopulmonary resuscitation and arthroscopic knee surgery, and subjects with various conditions, including end stage renal disease, cerebrovascular ischemia, acute mesenteric ischemia, systemic sclerosis, post-exercise skeletal muscle ischemia, diabetes mellitus, liver diseases and $\operatorname{PAD}(5,6)$.

Zimmerman et al. (12) stated that the response to ischemia in PAD is associated with the affected muscle mass, and a potent systemic response occurs only when there is proximal involvement affecting the entire extremity. In another study, it was found that serum IMA levels in patients with PAD decreased temporarily after exercise and returned to baseline levels within one hour (13).

Debashis et al. (13) found a relationship between postexercise skeletal muscle ischemia severity and IMA concentration, but stated that ischemia should develop acutely in order to alter IMA concentration. The lack of correlation between ABI values and IMA levels in the present study may be due to the absence of acute ischemia in these patients ( $\mathrm{p}>0.05)$.

\section{CONCLUSION}

The presence of diffuse lesions in patients with PAD is an indicator of poor prognosis. In order to predict the prognosis of these patients, there is a need for biomarkers besides results obtained with radiological techniques. Although no statistically significant relationship was 
found between lesion level and serum IMA level in this study, we believe that more comprehensive further studies may provide more informative data on this subject.

\section{ETHICAL DECLARATIONS}

Ethics Committee Approval: The study was carried out with the permission of Clinical Researches Ethics Committee of Okmeydanı Training and Research Hospital (Date: 19.06.2018, Decision No: 48670771-514.10).

Informed Consent: All patients signed the free and informed consent form.

Referee Evaluation Process: Externally peer-reviewed.

Conflict of Interest Statement: The authors have no conflicts of interest to declare.

Financial Disclosure: The authors declared that this study has received no financial support.

Author Contributions: All of the authors declare that they have all participated in the design, execution, and analysis of the paper, and that they have approved the final version.

\section{REFERENCE}

1. Hirsch AT, Haskal ZJ, Hertzer NR, et al. ACC/AHA 2005 Practice Guidelines for the management of patients with peripheral arterial disease (lower extremity, renal, mesenteric, and abdominal aortic): a collaborative report from the American Association for Vascular Surgery/Society for Vascular Surgery, Society for Cardiovascular Angiography and Interventions, Society for Vascular Medicine and Biology, Society of Interventional Radiology, and the ACC/AHA Task Force on Practice Guidelines (Writing Committee to Develop Guidelines for the Management of Patients With Peripheral Arterial Disease): endorsed by the American Association of Cardiovascular and Pulmonary Rehabilitation; National Heart, Lung, and Blood Institute; Society for Vascular Nursing; TransAtlantic Inter-Society Consensus; and Vascular Disease Foundation. Circulation 2006; 113: e463-e654.

2. Karabay Ö, Karaçelik M, Yllık L, et al. Ischemic peripheral arterial disease: A screening survey. Türk Gögüs Kalp Damar Cerr Derg 2012; 20: 450-7.

3. Küçükşen S, Toker A, Küçük A, et al. Evaluation of ischemia modified albumin levels and carotid intima media thickness in patients with systemic lupus eythematosus. Eur J Basic Med Sci 2014; 4: 77-82.

4. Ma SG, Wei CL, Hong B, Yu WN. Ischemia-modified albumin in type 2 diabetic patients with and without peripheral arterial disease. Clinics 2011; 66: 1677-80.

5. Sbarouni E, Georgiadou P, Voudris V. Ischemia modified albumin changes - review and clinical implications. Clin Chem Lab Med 2011; 49: 177-84

6. Piwowar A, Knapik-Kordecka M, Warwas M. Ischemia-modified albumin level in type 2 diabetes mellitus-Preliminary report. Dis Markers 2008; 24: 311-7.

7. Bar-Or D, Curtis G, Rao N, Bampos N, Lau E. Characterization of the $\mathrm{Co} 2+$ and $\mathrm{Ni2}+$ binding amino-acid residues of the $\mathrm{N}$ terminus of human albumin. Eur J Biochem 2001; 268: 42-8.

8. Ozkan U, Oguzkurt L, Tercan F. Atherosclerotic risk factors and segmental distribution in symptomatic peripheral artery disease. J Vasc Interv Radiol 2009; 20: 437
9. Diehm C, Kareem S, Lawall H. Epidemiology of peripheral arterial disease. Vasa. 2004; 33: 183-9

10. Cooke JP, Wilson AM. Biomarkers of peripheral arterial disease. J Am Coll Cardiol 2010; 55: 2017-23.

11. Bragadeesh T, Sari I, Pascotto M, Micari A, Kaul S, Lindner JR. Detection of peripheral vascular stenosis by assessing skeletal muscle flow reserve. J Am Coll Cardiol 2005; 45: 780-5.

12. Zimmerman BJ, Granger DN. Mechanisms of reperfusion injury. Am J Med Sci 1994; 307: 284-92.

13. Roy D, Quiles J, Sharma R, et al. Ischemia-modified albumin concentrations in patients with peripheral vascular disease and exercise-induced skeletal muscle ischemia. Clin Chem 2004; 50: 1656-60. 